\title{
Photoelectron spectroscopy method to reveal ionization potential lowering in nanoplasmas.
}

\author{
Beata Ziaja ${ }^{1,2, \star}$, Zoltan Jurek ${ }^{1,3}$, Nikita Medvedev ${ }^{1}$, Sang-Kil \\ Son ${ }^{1}$, Robert Thiele ${ }^{1}$, and Sven Toleikis ${ }^{4}$ \\ ${ }^{1}$ Center for Free-Electron Laser Science, Deutsches Elektronen-Synchrotron DESY, \\ Notkestrasse 85, D-22607 Hamburg, Germany \\ ${ }^{2}$ Institute of Nuclear Physics, Polish Academy of Sciences, Radzikowskiego 152, \\ 31-342 Kraków, Poland \\ ${ }^{3}$ On leave from Research Institute for Solid State Physics and Optics of the \\ Hungarian Academy of Sciences, 1525 Budapest P.O.Box 49, Hungary \\ ${ }^{4}$ Deutsches Elektronen-Synchrotron DESY, Notkestrasse 85, D-22607 Hamburg, \\ Germany \\ E-mail: ziaja@mail.desy.de
}

\begin{abstract}
Here we propose a scheme for probing the outer-shell atomic energy levels within a laser-created nanoplasma, using photoelectron spectroscopy data obtained from the irradiation of the nanoplasma with an ultraintense 'probing' pulse from soft X-ray free-electron laser. The proposed method can then detect shifts of outer-shell energy levels of an atom or an ion within a plasma, due to the effect of charged plasma environment on atomic potentials, known as the 'plasma screening effect'. Various theoretical models exist that estimate the magnitude of the screening effect. However, the first experimental data that can verify theoretical models have become available only recently (S. M. Vinko et al., Nature 482, 59 (2012)). They were obtained with a hard X-ray based experimental method which uses the information encoded in fluorescence spectra and is, therefore, restricted to deep atomic shells. Below we show that our photoelectron spectroscopy method of probing a nanoplasma with a destructive, high-intensity soft X-ray pulse that brings the irradiated system to the regime of 'massively parallel' ionization (Ch. Gnodtke, U. Saalmann, J.-M. Rost, Phys. Rev. Lett. 108, 175003 (2012)) enables the access to the information on the energy level (and ultimately energy level shift) of the valence orbital. In particular, the result of such photoelectron spectroscopy experiment could help clarifying the discrepancy between the ion abundances in nanoplasmas observed during the recent high-harmonic-generation and free-electron-laser experiments with intense soft X-ray pulses.
\end{abstract}

Keywords: Free-electron lasers; Plasma; Ionization potential lowering. 


\section{Introduction}

Recently developed X-ray free electron lasers (XFELs) such as FLASH [1], LCLS [2], SACLA[3], European XFEL [4], etc. can emit intense pulses of duration shorter or comparable with the characteristic times of the electron and ion dynamics within the irradiated system. This property of FEL radiation enables investigation of unique states of matter of femtosecond lifetimes, in particular studies of nanoplasmas created after irradiation of atomic clusters [5-13]. The dynamics within an irradiated cluster depends on the cluster size and pulse fluence. For small clusters the irradiation may lead to an efficient ionization of the whole cluster and its subsequent Coulomb explosion. Within large clusters two subsystems are formed: a net-neutral core and a positively charged outer shell. Thermalized electron plasma is quickly formed within the core; however, ions remain cold within the electron thermalization time. The electron plasma is stable on a timescale of a few up to several tens of femtoseconds, depending on the size of the cluster. Such system is especially convenient for studying the properties of electron plasma, in particular for studying the plasma screening effect on the atomic potentials.

Complementary to the FEL light sources, high-harmonic-generation (HHG) sources are developing rapidly, delivering femtosecond pulses of short-wavelength radiation, intense enough to create nanoplasmas from atomic clusters [14,15]. Interestingly enough, there is a discrepancy between findings of similar nanoplasma experiments performed with HHG and FEL sources. Using mass spectroscopy methods, HHG experiments detect high charge states at pulse fluences much lower than those ones used during FEL experiments. In other words, it seems that a HHG pulse of the same wavelength as a FEL pulse needs much less fluence $\left(\sim 7 \mathrm{~mJ} / \mathrm{cm}^{2}[15]\right)$ to create high charge states as compared to the fluence requirements of a similar experiment with FELs $\left(\sim 0.1 \mathrm{~J} / \mathrm{cm}^{2}[5]\right)$. An attempt to clarify this discrepancy was made in Ref. [15] by accounting for the specific effect of screening in electron nanoplasma: shifts of outershell atomic energy levels. The resulting lowering of ionization potential (IP) could lead to an efficient collisional ionization within the nanoplasma and to the generation of the observed high charge states [15].

However, the question arises how accurately we can estimate the IP lowering. There are many theoretical approaches to this problem. The shifts of energy levels within a single atom or ion due to the screening effect were first investigated by Ecker and Kroell (EK) [16] and then by Stewart and Pyatt (SP) [17]. Further calculations followed within the next years. Modifications of atomic properties in a plasma environment have been studied with the Debye screening model and the ion sphere model for oneelectron systems [18-20] and many-electron systems [21-24]. More recent calculations were performed by Hilse et al. [25] and Georgescu et al. [26] with nanoplasma models. Also, Gets and Krainov [27] calculated the ionization potentials for ions in rare gas clusters, using the Schrödinger equation with the Debye potential included. They found a larger deviation from the isolated atom case than that given by the Stewart-Pyatt shift. Other studies on lowering the ionization energy are summarized in Ref. [28] and, 
also in the context of electron-ion collision rates, in Refs. [29,30]. Recently, a study on extending the atomistic Hartree-Fock-Slater (HFS) model to include the screening induced by external charges has been performed by Thiele et al. [31].

Only recently, first experiments have been performed [32-34] that may allow for the verification of theoretical models by comparing them to data. An experimental collaboration led by J. Wark measured K-edge shifts and K-alpha emission from XFEL irradiated aluminum. The photon energies considered there were within the range of $1580-1720 \mathrm{eV}$. The K-edge position was estimated for charge states ranging from $\mathrm{Al}^{+3}$ to $\mathrm{Al}^{+7}$. Authors compared the experimental results with the predictions of the modified version of the FLYCHK code (SCFLY), corrected for the screening effects with the EK and SP models. They found their results in a better agreement with the predictions of the EK model than with the predictions of the SP model.

Here we propose another way of probing the screening effect, dedicated to investigate the IP lowering in nanoplasmas. This photoelectron spectroscopy method can probe the outer-shell atomic levels in nanoplasmas. These levels are not accessible with the currently available hard X-ray based method which uses the information encoded in fluorescence spectra and is, thus, 'per se' restricted to the inner shell levels. Therefore, our method can be considered as complementary to the hard X-ray based method, delivering the information on the modification of atomic potentials within their valence part. This method would then be suitable to detect lowering of ionization potentials within HHG created nanoplasmas.

\section{Probing the nanoplasma with an ultraintense, ultrashort soft X-ray FEL pulse}

In the following theoretical study we consider nanoplasmas created from irradiated argon clusters. These weakly-coupled atomic clusters are chosen in order to avoid the influence of the initial interatomic bonds on the atomic energy levels. The photon energy of the probe pulse lies within the soft X-ray regime, below the Ar core ionization threshold ( $250 \mathrm{eV}$ for $\mathrm{L}$ shell). Electron emission from such irradiated clusters have been extensively studied both experimentally $[14,15,35]$ and theoretically [36-38].

Let us consider a nanoplasma created from a soft X-ray irradiated argon cluster. It can be created with currently operating light sources, e.g. after irradiation with HHG pulse (21st harmonic from intense NIR femtosectond laser $\sim 38 \mathrm{~nm}$ ) or soft X-ray FEL. Efficiency of both schemes has been demonstrated experimentally $[7,8,14,15,35$, $39,40]$. In our study we investigate the state of the nanoplasma created from Ar cluster consisting of 10000 atoms after its irradiation with a rectangular laser pulse of photon energy, $E=41 \mathrm{eV}$ (30 nm wavelength), pulse duration, 5 fs, and intensity, $5 \cdot 10^{14} \mathrm{~W} / \mathrm{cm}^{2}$. This corresponds to the total peak pulse fluence of $2.5 \mathrm{~J} / \mathrm{cm}^{2}$. Pulse duration has been chosen so short in order to simplify the computational effort during simulations, as within this linear photoabsorption regime the final state of the sample (i.e. its ionization degree, electron temperature etc.) depends only on the fluence of the plasma-creating 
pulse and not on the temporal characteristics of the pulse. Experimentally, HHG pulses of $\sim 10$ fs duration are feasible [14]. During an experiment one can then use such pulses and adjust their intensity so as to obtain the desired pulse fluence, necessary to create a nanoplasma of similar parameters as considered above.

Let us note that at this soft X-ray photon energy the dominant ionization mechanism of the cluster is the sequential ionization $[7,38]$. The heating of electrons due to the inverse bremsstrahlung (IB) process is negligible. Photoelectrons released by $41 \mathrm{eV}$ soft X-ray photons from Ar atoms through the dominant photoionization channel have the energy of $\sim 25 \mathrm{eV}$. They can leave the irradiated system within a few fs, forming a characteristic photoelectron peak within the electron spectra. The net charge of the system then starts to increase, and prevents the escape of further electrons from the cluster. These trapped electrons can cause further secondary ionizations. They also interact with each other which leads to a fast electron thermalization on fs timescale. It is energetically preferred for the trapped electrons to move towards the center of the sample, forming a net-neutral core with ions. This leads to the formation of a positively charged outer shell of ions around the neutral core. For samples large enough and sufficiently highly ionized, even high-energy photoelectrons can be trapped within the core, enhancing the temperature of the quasi-free electrons. However, it should be noted that ions stay cold during this irradiation, as the plasma-creating pulse is very short ( $\sim$ few fs) compared to the electron-ion equilibration time ( $\sim$ few ps). Because of screening by trapped electrons, only the outer shell ions see a radial Coulomb force $[41,42]$. For a large cluster, the number of ions within this positively charged outer shell is small, comparing with the number of ions within the net-neutral cluster core. As the result, after the short soft X-ray pulse, the initially neutral large cluster evolves in principle into a two-regime system consisting of cold ions located within the interior of the cluster, embedded in a hot thermalized free-electron plasma.

The theoretical simulations were performed with the non-equilibrium Boltzmann code $[43,44]$, solving the coupled kinetic evolution equations for electron and ion distributions $[43,44]$. The version of the Boltzmann code used for this specific simulation included the following processes: photo- and collisional (impact) ionization, three-body recombination, elastic electron-ion and electron-atom scattering, and shortrange electron-electron interactions. Direct multiphoton ionization is secondary at the considered pulse intensities $[45,46]$ and was neglected here. Also, the heating of electrons due to the inverse bremsstrahlung process is negligible. The kinetic equations are solved on a grid within the simulation box that contains the Ar cluster, located in the center. The radial size of the box is a few times larger than the cluster radius. In this way the particles leaving the cluster can be followed. The processes listed above can occur anywhere within the simulation box (not only within the cluster), depending on the actual interaction rates, estimated on-the fly. Let us emphasize that in order not to bias our analysis by a specific choice of the screening model, during the simulations we do not shift the atomic energy levels in the presence of plasma. In what follows we aim to investigate the emitted electron spectrum in order to identify constraints for the 
detectability of the atomic energy levels (and ultimately, their shifts).

Various simulation parameters and cluster size (200 - 10000 atoms) were tested in order to achieve a sufficiently strong signal within the electron spectra after the probe pulse. The results of our simulations for $A r_{10000}$ cluster are shown in Fig. 1. They confirm fast electron thermalization with the final temperature of $\sim 12 \mathrm{eV}$ and the creation of stable electron plasma conditions (in local thermodynamic equilibrium (LTE)) within a few femtoseconds after the irradiation. The efficient trapping of photoelectrons starts after $\geq 2$ fs. At this time the kinetic electron temperature reaches its minimal value, and then again starts to increase. Time-integrated electron spectra indicate the position of the primary photoelectron peak at $\sim 25 \mathrm{eV}$ (visible as a bump on the tail of thermal distribution), corresponding to the ionization of neutral Ar atoms (Fig. 1c). At lower electron energies the characteristic thermal distribution is formed [38]. Atoms $\mathrm{Ar}^{+0}$ (65\%) and ions $\mathrm{Ar}^{+1}(30 \%), \mathrm{Ar}^{+2}(5 \%)$ can be found within the sample. However, the structure of the corresponding photoelectron peaks is not well visible as the peaks become 'dissolved' within the dominating thermal distribution of low-energy electrons.

The created nanoplasma has been probed with another intense, ultrashort, soft Xray pulse (Fig. 2). Similar pump-probe schemes with ultrashort laser pulses have been proposed in Refs. $[47,48]$ for the purpose of investigating the non-equilibrium plasma properties. Here we use the intense 'probe' pulse to estimate the ionization potential of Ar atoms and ions within the equilibrated electron plasma. For this purpose we have to use a sufficiently high 'probe' photon energy, yielding the secondary photoelectron energy a few times larger than that one of the primary photoelectron. Otherwise, the secondary photoelectron peaks will be not visible as their positions will overlap with the primary ones and with the thermal electron distribution. The photon energy used for 'probing' during this simulation was $E=123 \mathrm{eV}$, corresponding to the radiation wavelength of $10 \mathrm{~nm}$. This is experimentally feasible with FELs. We checked through dedicated atomic calculations that photons at this energy predominantly excite outershell photoelectrons from Ar atoms and ions.

Below we show the results obtained from probing the nanoplasma (Fig. 2c-d). In our simulations, the rectangularly shaped probing pulse of intensity, $5 \cdot 10^{16} \mathrm{~W} / \mathrm{cm}^{2}$, irradiates the nanoplasma between $5-10$ fs. The time integration extends over 40 fs since the start of the exposure to the first, plasma-creating soft X-ray pulse. This is long enough, as after 40 fs only a small number of free electrons are left within the probed cluster, so no further electron emissions will occur after this time. As the conditions of the created LTE electron plasma seem stable on tens femtosecond timescale (Fig. 1), similar probe results can be expected if delaying the probe by a few tens of femtoseconds since the end of the plasma-creating pulse. This imposes a constraint on the temporal synchronisation of the plasma-creating and plasma-probing pulses during the experiment, i.e. the 'probe' pulse should arrive while electron nanoplasma conditions are stable. Usually, the time jitter between NIR and FEL pulse can be $<100$ fs r.m.s. This implies that during the repeated many-shot experiment some FEL pulses can arrive before this time, i.e. still within the stability regime of large nanoplasmas. These events 
could then be identified with a novel technique of retrieving the information on the arrival of individual FEL pulses on a time base synchronized to the external laser to within 6 fs r.m.s. [49].

Photoelectron spectroscopy delivers the information on the photoelectrons produced within the cluster that were able to leave the irradiated cluster. After the pump pulse the $\operatorname{Ar}_{10000}$ cluster consists of $\operatorname{Ar}^{+0}$ (65\%), $\mathrm{Ar}^{+1}$ (30\%) and $\mathrm{Ar}^{+2}$ (5\%). Our test simulations showed that only after irradiating this cluster with a high intensity probe pulse some photoelectrons - created then via massively parallel ionization [50]- can leave the cluster. However, these are only the photoelectrons from the photoionization of neutral $\mathrm{Ar}^{+0}$ to $\mathrm{Ar}^{+1}$ by probe pulse that show up in the emitted electron spectra. Snapshots of the photoelectron density recorded outside the cluster (not shown) confirm the formation of a photoelectron 'wave' within 1 fs of the exposure to the probe pulse. This collective wave of the maximum energy at $107 \mathrm{eV}$ results from the probe pulse photoproduction of electrons in the process, $\mathrm{Ar}^{+0}$ to $\mathrm{Ar}^{+1}$. It then leads to the formation of the secondary photoelectron peak in the emitted electron spectra. After this time the positive charge inside the sample increases so significantly that less energetic photoelectrons produced by probe pulse photoionization of $\mathrm{Ar}^{+1}, \mathrm{Ar}^{+2}$ are not able to leave the sample collectively but only through evaporation. The same occurs after the photoionization of higher charges $\mathrm{Ar}^{+3}-\mathrm{Ar}^{+5}$.

Let us mention that this secondary (probe) photoelectron emission still shows similarities to the primary (pump) photoelectron emission initiated by the lower pulse fluence. Only the photoelectrons from the process, $\mathrm{Ar}^{+0}$ to $\mathrm{Ar}^{+1}$, emitted from the initially neutral cluster form the visible primary photoelectron peak within the emitted electron spectra. Photoelectrons emitted from $\mathrm{Ar}^{+1}, \mathrm{Ar}^{+2}$ are slowed down or even confined by the positive field inside the ionized cluster and are not visible within the emitted electron spectra.

The high intensity probe pulse allows the probe-excited photoelectrons to overcome the attracting field of the cluster after the pump pulse, and to form the secondary photoelectron peak from the photoionization process, $\mathrm{Ar}^{+0}$ to $\mathrm{Ar}^{+1}$. However, the contribution of further secondary photoionizations to the photoelectron spectra is strongly suppressed due to the extensively growing space charge.

Massively parallel ionization, first described in Ref. [50], requires high intensity of the 'probe' laser of $\sim 10^{16} \mathrm{~W} / \mathrm{cm}^{2}$ (Fig. 2d). At such high intensity photoelectrons are created very fast and can leave the sample almost simultaneously. They screen collectively the increasing positive cluster charge but also interact strongly with each other [50]. Characteristic broadening of photoelectron spectra was explained in Ref. [50] as the result of the convolution of the mean-field result with the spectrum governed by binary short-range electron-electron collisions. In our Boltzmann model we treat both long-range (mean-field) and short-range electron-electron interactions so this effect is also taken into account.

In view of these arguments, we can exclude any significant contribution of photoelectrons emitted after the photoionization of $\mathrm{Ar}^{+1}$ and higher charges to the 
secondary photoionization peak and interpret the specific shape of this peak as the effect of broadening due to the short-range photoelectron-photoelectron collisions (cf. Fig. 2 in [50]). The maximum of the secondary peak is well distinguishable and corresponds to the energy of $\sim 102 \mathrm{eV}$ (fitted with a Gaussian fit with the accuracy of $\sim 0.1 \%$ ). The FWHM of the Gaussian peak is $\sim 37 \mathrm{eV}$. The difference between the expected photoelectron energy of $107 \mathrm{eV}$ and the position of the maximum of Gaussian fit is 5 $\mathrm{eV}$. This shift can be explained by the space charge effect and the broadening effect of short-range electron-electron interactions. It gives a rough estimate for the accuracy of the detection of the valence energy levels in this specific case. The shift of the valence level due to the plasma screening can be detectable, if it is larger than $5 \mathrm{eV}$.

As mentioned above, in order to avoid a bias of a specific screening model, in the simulations we assumed no IP lowering due to the plasma screening. The predictions for the IP lowering obtained with the extended HFS code [31] at the actual plasma conditions would place the IP shift between 5-10 eV for the $\mathrm{Ar}^{+0}$. Such shift could then be detectable in the 'massively parallel' photoelectron spectra.

\section{Conclusions}

The purpose of this work was to investigate if the information on the shifts of outer-shell atomic energy levels due to the plasma screening can be extracted from the photoelectron spectra recorded after probing a nanoplasma with an ultrashort soft X-ray pulse. We have shown that the access to the information on the shifts can be achieved by probing the nanoplasma with a destructive, high-intensity pulse, during which the system enters the regime of the massively parallel ionization. The secondary photoelectron peak becomes then visible with an energy resolution that would allow to identify an IP lowering of $\geq 5 \mathrm{eV}$ for the nanoplasma created from $A r_{10000}$ cluster. We hope that our analysis may inspire further theoretical and experimental efforts with the combined HHG and FEL short-wavelength light sources, using the photoelectron spectroscopy for investigation of the IP lowering in nanoplasmas. Such experiments seem feasible: from the technical point of view their most difficult part would be the installation of HHG source at the beamline at FLASH facility at DESY. This is a non-standard operation which would require extra time. Because of time reasons it could then not be performed within a standard beamtime granted by facility but it would require a dedicated study beamtime.

\section{Acknowledgements}

We thank A. Przystawik and R. Santra for illuminating comments and discussions.

\section{References}

[1] W. Ackermann et al. Nat. Phot., 1:336, 2007.

[2] P. Emma et al. Nature Photonics, 4:641-644, 2010. 
(a)

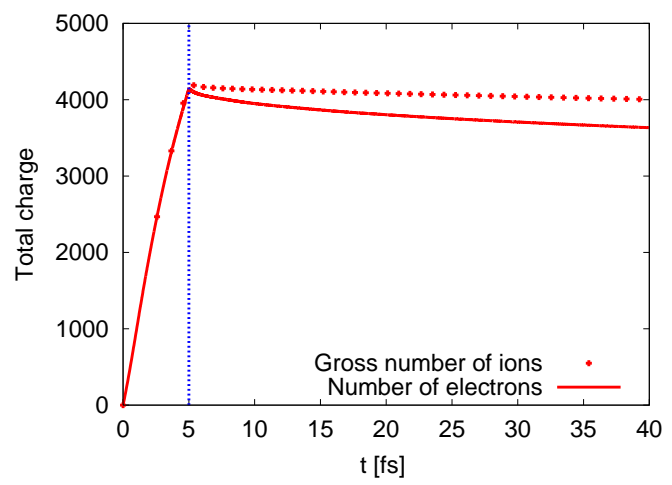

(c)

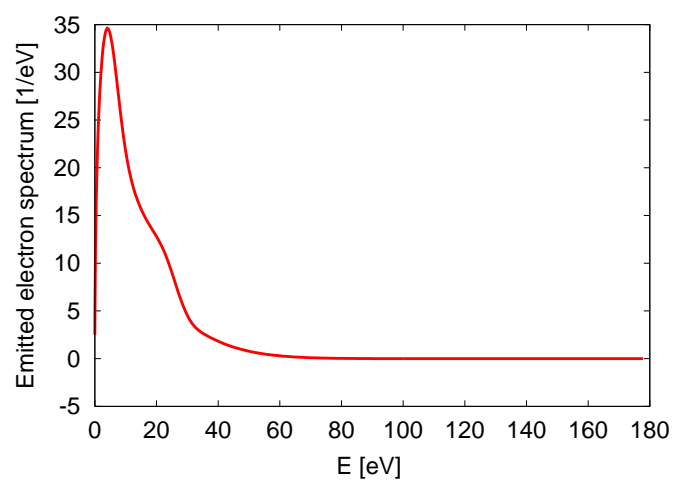

(b)
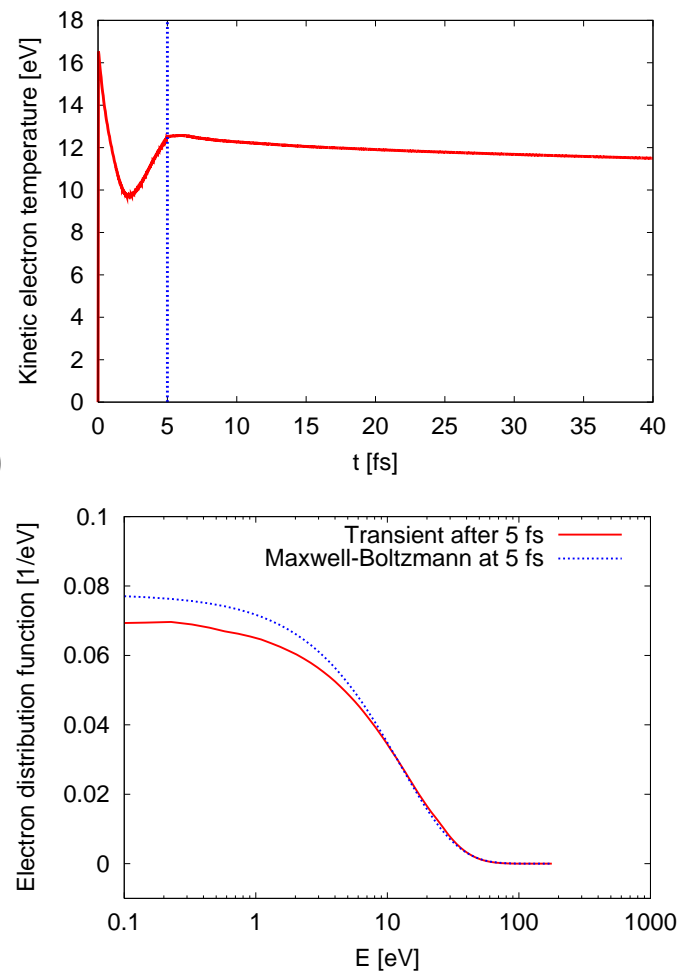

Figure 1. Nanoplasma created from $A r_{10000}$ cluster irradiated by soft X-ray FEL pulse of wavelength $30 \mathrm{~nm}$, duration $5 \mathrm{fs}$, and intensity $5 \cdot 10^{14} \mathrm{~W} / \mathrm{cm}^{2}$ : (a) time evolution of ion charge represented by the gross number of ions, i.e. the number of ions of a given charge multiplied by the number charge, summed up over all charge states within the system (points) and electron charge (lines) within the cluster, (b) electron temperature as a function of time, (c) emitted electron spectrum, time-integrated over 40 fs since the start of the exposure, and (d) thermalized transient electron distribution within the cluster after $5 \mathrm{fs}$, compared to the Maxwell-Boltzmann distribution calculated with the model results: electron kinetic temperature and electron density in the cluster center. In (a-b) the end of the plasma-creating (rectangularly-shaped) pulse is indicated by the vertical line.

[3] T. Ishikawa et al. Nat. Phot., 6:540-544, 2012.

[4] M. Altarelli et al. in: The European X-Ray Free-Electron Laser Technical Design Report. DESY: Hamburg, 2007.

[5] H. Wabnitz et al. Nature, 420:482, 2002.

[6] T. Laarmann et al. Phys. Rev. Lett., 95:063402, 2005.

[7] C. Bostedt et al. Phys. Rev. Lett., 100:133401, 2008.

[8] H. Thomas et al. J. Phys. B, 42:134018, 2009.

[9] R. Santra and C. H. Greene. Phys. Rev. Lett., 91:233401, 2003.

[10] C. Siedschlag and J.M. Rost. Phys. Rev. Lett., 93:043402, 2004.

[11] C. Jungreuthmayer et al. J. Phys. B, 38:3029, 2005.

[12] D. Bauer. J. Phys. B, 37:3085, 2004.

[13] B. Ziaja, H. Wabnitz, F. Wang, E. Weckert, and T. Möller. Phys. Rev. Lett., 102:205002, 2009.

[14] B. F. Murphy et al. Phys. Rev. Lett., 101:203401, 2008.

[15] K. Hoffmann et al. Phys. Rev. A, 83:043203, 2011.

[16] G. Ecker and W. Kroell. Phys. Fluids, 6:62, 1963. 
(a)
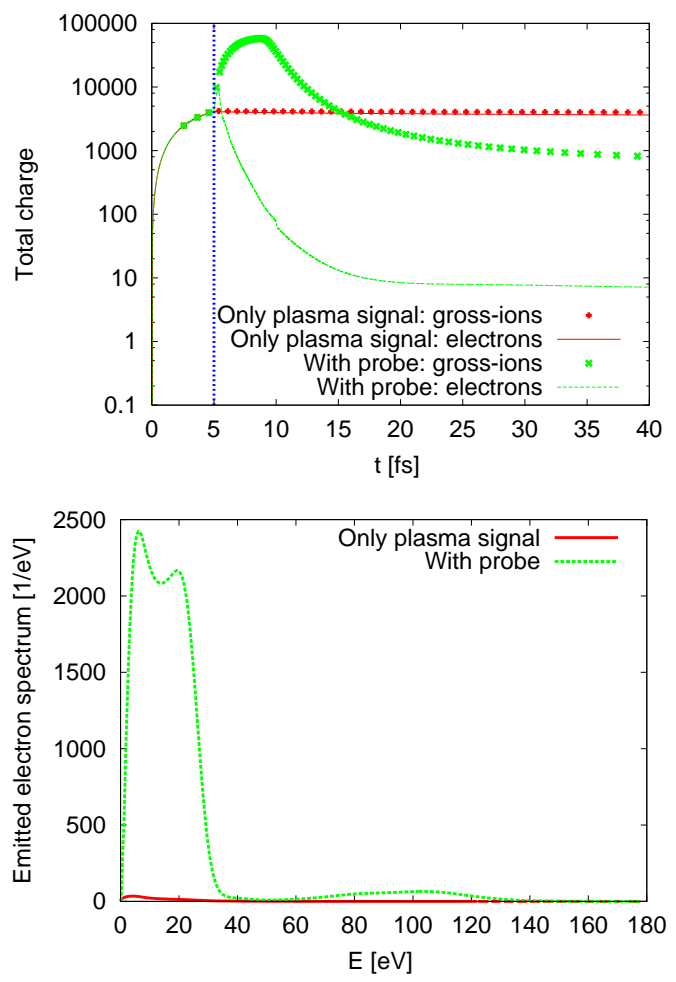

(b)
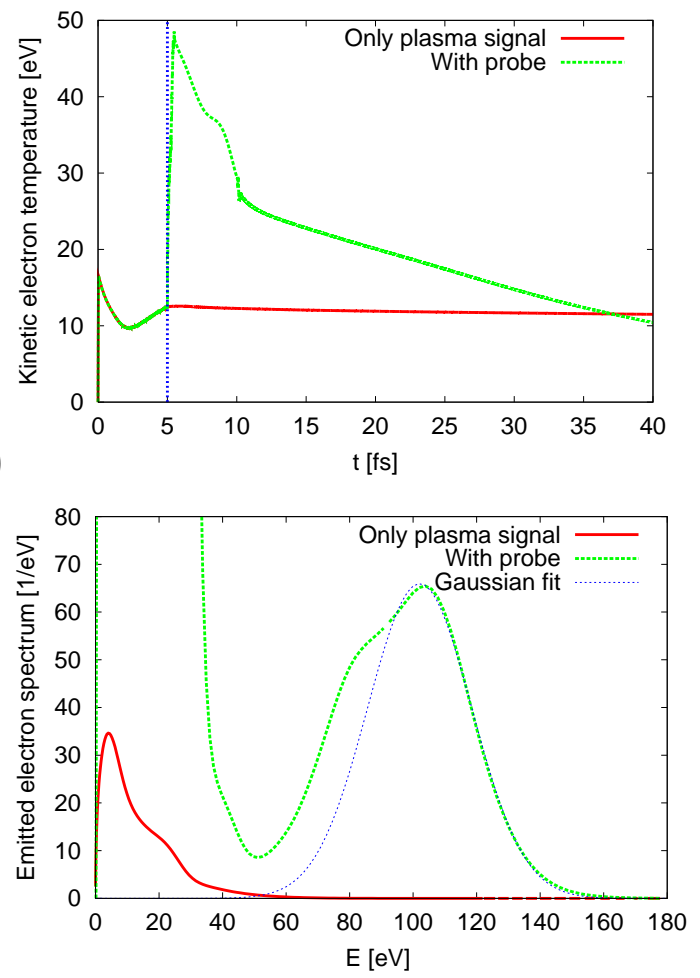

Figure 2. Nanoplasma probed with an intense soft X-ray FEL pulse: (a-b) plasma parameters as a function of time (notation as in Fig. 1), and (c-d) time-integrated spectra of emitted electrons, obtained after irradiating a nanoplasma with an intense FEL pulse of wavelength of $10 \mathrm{~nm}$, duration of $5 \mathrm{fs}$, and intensity $5 \cdot 10^{16} \mathrm{~W} / \mathrm{cm}^{2}$. The time integration extends over $40 \mathrm{fs}$ since the start of the exposure to the plasma-creating soft X-ray pulse. Part (d) shows the details of the secondary photoelectron peak from the electron emission spectra (c), including the Gaussian fit. For reference the creation of nanoplasma is also shown (up to $5 \mathrm{fs}$ ), as well as the undisturbed plasma evolution ('Only plasma signal'). In (a-b) the end of the plasma-creating pulse is indicated by the vertical line. Later, the rectangularly shaped probing pulse irradiates the nanoplasma between $5-10$ fs ('With probe').

[17] J.P. Stewart and K.D. Pyatt. Astrophys. J., 144:1203, 1966.

[18] S. Bhattacharyya, A. N. Sil, S. Fritzsche, and P. K. Mukherjee. Eur. Phys. J. D, 46:1, 2008.

[19] B. Saha, P. K. Mukherjee, and G. H. F. Diercksen. A\&A, 396:337, 2002.

[20] J.Saha et al. Eur. Phys. J. D, 62:205, 2011.

[21] P. K. Mukherjee, J. Karwowski, and G. H. F. Diercksen. Chem. Phys. Lett., 363:323, 2002.

[22] B. Saha, T.K. Mukherjee, P.K. Mukherjee, and G.H.F. Diercksen. Theor. Chem. Acc., 108:305, 2002.

[23] M. Das et al. J. Phys. B: At. Mol. Opt. Phys., 44:165701, 2011.

[24] M. Das et al. Phys. Rev. A, 85:042506, 2012.

[25] P. Hilse, M. Moll, M. Schlanges, and Th. Bornath. Laser Physics, 19:428-436, 2009.

[26] I. Georgescu, U. Saalmann, and J.-M. Rost. Phys. Rev. A, 76:043203, 2007.

[27] A. V. Gets and V. P. Krainov. J. Phys. B At. Mol. Opt. Phys., 39:1787, 2006.

[28] M. S. Murillo and J. C. Weisheit. Physics Reports, 302:1, 1998.

[29] M. Moll et al. J. Phys. B: At. Mol. Opt. Phys., 43:135103, 2010. 
[30] M. Moll et al. Phys. Plasmas, 19:033303, 2012.

[31] R. Thiele, S.-K. Son, B. Ziaja, and R. Santra. Phys. Rev. A, 86:033411, 2012.

[32] S.M. Vinko et al. Nature, 482:59-62, 2012.

[33] O. Ciricosta et al. Phys. Rev. Lett., 109:065002, 2012.

[34] B. I. Cho et al. Phys. Rev. Lett., 109:245003, 2012.

[35] Ch. Bostedt et al. Phys. Rev. Lett., 108:093401, 2012.

[36] Ch. Gnodtke, U. Saalmann, and J.-M. Rost. New J. Phys., 13:013028, 2011.

[37] Ch. Gnodtke, U. Saalmann, and J.-M. Rost. Chem. Phys., in press, 2012.

[38] B. Ziaja et al. New J. Phys., 11:103012, 2009.

[39] S. Schorb et al. Phys. Rev. Lett., 108:233401, 2012.

[40] C. Bostedt et al. New J. Phys., 12:083004, 2010.

[41] S. P. Hau-Riege, R. A. London, and A. Szoke. Phys. Rev. E, 69:051906, 2004.

[42] Z. Jurek and G. Faigel. Eur. Phys. J. D, 50:35, 2008.

[43] B. Ziaja, H. Wabnitz, E. Weckert, and T. Möller. New J. Phys, 10:043003, 2008.

[44] B. Ziaja, H. Wabnitz, E. Weckert, and T. Möller. Europhys. Lett., 82:24002, 2008.

[45] G. Doumy et al. Phys. Rev. Lett., 106:083002, 2011.

[46] A. Sytcheva, S. Pabst, S.-K. Son, and R. Santra. Phys. Rev. A, 85:023414, 2012.

[47] J.-M. Rost. European Review, 17:249, 2009.

[48] U. Saalmann, I. Georgescu, and J.-M. Rost. New J. Phys., 10:025014, 2008.

[49] I. Grguras et al. Nat. Phot., 6:852, 2012.

[50] Ch. Gnodtke, U. Saalmann, and J.-M. Rost. Phys. Rev. Lett., 108:175003, 2012. 